\title{
Cortico-striatal spike-timing dependent plasticity after activation of subcortical pathways
}

\author{
Jan M. Schulz ${ }^{*}$, Peter Redgrave ${ }^{2}$ and John N. J. Reynolds ${ }^{1}$ \\ Department of Anatomy and Structural Biology, School of Medical Sciences, University of Otago, Dunedin, New Zealand \\ Department of Psychology, University of Sheffield, Western Bank, Sheffield, UK
}

Edited by:

Henry Markram, Ecole Polytechnique Federale de Lausanne, Switzerland

\section{Reviewed by:}

Guo-Qiang Bi, University of Pittsburgh, USA

Henry Markram, Ecole Polytechnique

Federale de Lausanne, Switzerland

\section{${ }^{*}$ Correspondence}

Jan M. Schulz, Department of Anatomy and Structural Biology, University of Otago, P.O. Box 913, Dunedin 9054, New Zealand.

e-mail: jan.schulz@anatomy.otago. ac.nz
Cortico-striatal spike-timing dependent plasticity (STDP) is modulated by dopamine in vitro. The present study investigated STDP in vivo using alternative procedures for modulating dopaminergic inputs. Postsynaptic potentials (PSP) were evoked in intracellularly recorded spiny neurons by electrical stimulation of the contralateral motor cortex. PSPs often consisted of up to three distinct components, likely representing distinct cortico-striatal pathways. After baseline recording, bicuculline $(\mathrm{BIC})$ was ejected into the superior colliculus (SC) to disinhibit visual pathways to the dopamine cells and striatum. Repetitive cortical stimulation $(\sim 60 ; 0.2 \mathrm{~Hz})$ was then paired with postsynaptic spike discharge induced by an intracellular current pulse, with each pairing followed $250 \mathrm{~ms}$ later by a light flash to the contralateral eye $(n=13)$. Changes in PSPs, measured as the maximal slope normalized to 5-min pre, ranged from potentiation $(\sim 120 \%)$ to depression ( $80 \%)$. The determining factor was the relative timing between PSP components and spike: PSP components coinciding or closely following the spike tended towards potentiation, whereas PSP components preceding the spike were depressed. Importantly, STDP was only seen in experiments with successful BIC-mediated disinhibition $(n=10)$. Corticostriatal high-frequency stimulation $(50$ pulses at $100 \mathrm{~Hz}$ ) followed $100 \mathrm{~ms}$ later by a light flash did not induce more robust synaptic plasticity $(n=9)$. However, an elevated post-light spike rate correlated with depression across plasticity protocols $\left(R^{2}=0.55, p=0.009, n=11\right.$ active neurons). These results confirm that the direction of cortico-striatal plasticity is determined by the timing of pre- and postsynaptic activity and that synaptic modification is dependent on the activation of additional subcortical inputs.

Keywords: STDP, striatum, superior colliculus, spiny projection neuron, HFS, in vivo, intracellular, dopamine

\section{INTRODUCTION}

The striatum, the main input nucleus of the basal ganglia, receives sensorimotor, cognitive, and motivational information from almost all cortical areas (McGeorge and Faull, 1989) and several thalamic nuclei (Erro et al., 2002). In addition, evaluative inputs from dopaminergic neurons in the substantia nigra pars compacta (Redgrave et al., 2008; Wickens et al., 2007a) and glutamatergic neurons in the thalamic intralaminar nuclei (Minamimoto et al., 2005; Schulz et al., 2009) signal the saliency of ongoing sensory events to broad areas of the striatum. The convergence of these different sets of inputs places the striatum in an ideal position for learning guided by experience. Based on anatomy and physiology, it is likely that the underlying mechanisms are dopaminedependent (Wickens et al., 2007a,b). Thus, synaptic plasticity in the striatum is strongly modulated by dopamine (Wickens et al., 1996; Reynolds and Wickens, 2000; Kerr and Wickens, 2001; Pawlak and Kerr, 2008; Shen et al., 2008). Furthermore, the stimulation of the substantia nigra in intracranial self-stimulating experiments is behaviorally reinforcing and the same stimulation parameters induce robust potentiation of cortico-striatal synapses (Reynolds et al., 2001). These observations indicate that dopamine is a critical ingredient for modulating cortico-striatal neurotransmission.
A major problem in investigating the functional role of dopamine signals for synaptic plasticity is that it has been technically challenging to manipulate its levels in a physiological meaningful way. In the present study, therefore, we deployed procedures that have been shown previously to evoke the release of dopamine by more natural means (Coizet et al., 2003; Comoli et al., 2003; Dommett et al., 2005). In urethane anesthetized rats, disinhibition of the superior colliculus (SC) by a local injection of the GABA antagonist bicuculline (BIC) enables visual-evoked activation of dopaminergic neurons. Thus, a visual event can activate dopaminergic neurons via a subcortical pathway from retinal ganglion cells through the SC. In parallel, a tecto-thalamo-striatal pathway mediates excitatory inputs that rapidly depolarize spiny projection neurons (SPN), the principal neurons of the striatum, in response to the same sensory event (Schulz et al., 2009). Therefore, for the first time it was possible to control these subcortical inputs to the striatum using a physiological stimulus during intracellular recordings from SPNs.

This study investigated how visual-evoked inputs from tectostriatal pathways affected the outcome of conventional plasticity protocols on the cortico-striatal synapse. The visual stimulus was delivered at a short, physiological meaningful delay as suggested by theoretical work (Izhikevich, 2007) and our previous experience (Reynolds et al., 2001). First, we measured cortico-striatal 
synaptic efficacy after cortico-striatal high-frequency stimulation (HFS) paired with visual stimulation. Previously, we reported that HFS induced synaptic depression in vivo that could be blocked or reversed by concomitant electrical stimulation of dopaminergic neurons (Reynolds and Wickens, 2000). In this study, we tested the effects of a more physiological activation of afferent networks. Next, a spike-timing dependent plasticity (STDP) protocol involving pairing of single pre- and postsynaptic spikes in the corticostriatal pathway was tested in combination with visual activation. Although STDP has been demonstrated at the cortico-striatal synapse in vitro, there is controversy as to whether a pre-post pairing protocol induces depression or potentiation (Fino et al., 2005; Pawlak and Kerr, 2008). The present study aimed at clarifying this issue in an intact in vivo model.

\section{MATERIALS AND METHODS EXPERIMENTAL PREPARATION}

Every effort was made to minimize the pain and discomfort of the experimental animals used in this study, in accordance with approvals granted by the University of Otago Animal Ethics Committee. Hundred and forty male Long-Evans rats (230-410 g) were anesthetized with urethane (1.4-1.9 g/kg i.p.; Biolab Ltd., Auckland, New Zealand), supplemented with additional urethane $(0.2 \mathrm{~g} / \mathrm{kg})$ one to two-hourly, as required. The core temperature was maintained at $36^{\circ} \mathrm{C}$ by a homoeothermic blanket. All wounds and pressure points were infiltrated with a long-acting local anesthetic (bupivacaine, $0.5 \%)$. The head was fixed in a stereotaxic frame and surgery was performed as previously described (Schulz et al., 2009). Briefly, a hole was drilled above the left SC, around $6.5 \mathrm{~mm}$ posterior bregma (AP -6.5$)$ and $1.4 \mathrm{~mm}$ mediolateral from the midline $(\mathrm{ML}+1.4)$, to accept a pipette pulled from pre-calibrated glass capillaries (volume $5 \mu \mathrm{l}$, diameter $1.0 \mathrm{~mm}$; Modulohm I/S, Denmark) and filled with BIC (0.01\% in saline; Sigma). The BIC ejection pipette was lowered $4.1 \mathrm{~mm}$ from brain surface into the SC deep layers and secured in place with dental cement. To document the restoration of visual sensitivity enabled by BIC (Coizet et al., 2003; Dommett et al., 2005), the local field potential (LFP) was routinely recorded in the SC deep layers through an attached recording electrode. Above the right motor cortex, a hole was drilled around AP +2.0/ ML -1.8 to accept concentric electrodes (NEX-100, Rhodes, USA). Stimulating electrodes were secured in place at a depth of 1.8 to $2 \mathrm{~mm}$. A craniotomy from AP -2.0 to +2.5 and $\mathrm{ML}+0.5$ to +4.0 was made to provide access to the left medial striatum.

\section{INTRACELLULAR RECORDINGS}

Intracellular records were made using micropipettes pulled from 3.0-mm diameter glass (Harvard Apparatus, UK) and filled with $1 \mathrm{M} \mathrm{K}$-acetate (45 to $95-\mathrm{M} \Omega$ resistance), in some cases containing $2 \%$ biocytin. The micropipette was advanced through the striatum from initial penetrations between $\mathrm{AP}-0.1$ to +1.6 and $\mathrm{ML}$ +2.6 to +4.0 , until a stable recording was obtained from a putative SPN. These coordinates were chosen to maximize the chance that recorded neurons would receive direct innervation from the contralateral motor cortex that was stimulated (Wilson, 1987; McGeorge and Faull, 1989). All electrophysiologically identified SPNs exhibited: (i) fluctuations of $>7 \mathrm{mV}$ in amplitude between a hyperpolarized Down state and a depolarized Up state (Wilson and Kawaguchi, 1996), (ii) a Down state membrane potential more negative than $-70 \mathrm{mV}$, and (iii) a slow ramp-like depolarization in response to a just suprathreshold current pulse (see Figure 1), as typical for these neurons (Nisenbaum and Wilson, 1995; Mahon et al., 2000; Reynolds and Wickens, 2000). Current-voltage relations were obtained by injecting hyperpolarizing and depolarizing current pulses through the micropipette during the neuron's Down state, using an Axoclamp-2B amplifier (Axon Instruments Inc., Union City, CA, USA) configured in current-clamp mode. Input resistance was determined from the slope of a regression line fitted to four membrane potentials produced by a series of subthreshold current pulses $(-0.2,0,+0.2,+0.4 \mathrm{nA})$.

\section{STIMULATION OF AFFERENT PATHWAYS}

Biphasic rectangular stimulus pulses of 100 to 200 - $\mu$ s duration were applied to the contralateral motor cortex to evoke cortico-striatal postsynaptic potentials (PSPs) in SPNs. Stimulus intensity $(<1 \mathrm{~mA})$ and width were chosen to produce a PSP of 5 to $10 \mathrm{mV}$ in amplitude. Except for group 3 (see below), test stimuli were applied during a recorded neuron's Down state. Up to Down state transitions were detected using a locally-constructed threshold discriminator (Reynolds and Wickens, 2003). Detected transitions were used to trigger data acquisition and cortical stimulation after a 25-ms delay. All waveform data were digitized at $10 \mathrm{kHz}$ by a Digidata $1322 \mathrm{~A}$ (Axon Instruments Inc.), displayed using pClamp 10 software (Axon Instruments Inc.) and stored to disk. The baseline recording consisted of at least 50 responses to test stimuli at $0.1 \mathrm{~Hz}$ (group 1 and 2) or $0.2 \mathrm{~Hz}$ (group 3 and 4) recorded over $15 \min (n=68$ neurons recorded over 2 years). Following baseline recording, BIC (200-300 nl) was ejected into the SC at a rate of $\sim 200 \mathrm{nl} / \mathrm{min}$. Striatal membrane potential activity was recorded directly after the BIC ejection, before cortical stimulation was paired repetitively with a light stimulus (1500 mcd, 10-ms duration) from a white LED positioned $<3 \mathrm{~cm}$ directly in front of the animal's right eye. After the plasticity protocol, single pulse stimulation was resumed ( 0.1 or $0.2 \mathrm{~Hz}$ ).

\section{PLASTICITY PROTOCOLS}

Four different pairing protocols were used (Figure 2). In experiments involving HFS, stimulation was always triggered at Down state membrane potentials. Because of BIC-induced loss of EEG slow-wave activity (Schulz et al., 2009), the inter-HFS train intervals without data acquisition varied from 10 to $60 \mathrm{~s}$. Therefore, the period of the plasticity protocol (of $\sim 5 \mathrm{~min}$ ) is not included in the time-resolved membrane potential distribution in Figure 3.

\section{Group 1: HFS+ spikes then light}

High-frequency stimulation (six trains of 50 pulses at $100 \mathrm{~Hz}$ ) of the cortex was paired with a just suprathreshold depolarizing current pulse ( 0.5 to $0.9 \mathrm{nA}, 570 \mathrm{~ms}$ ). Each train was followed by a light stimulus at a delay of $100 \mathrm{~ms}$ after the cessation of the HFS.

\section{Group 2: HFS then light+ spikes}

During the HFS protocol (six trains of 50 pulses at $100 \mathrm{~Hz}$ ), the suprathreshold current step ( 0.5 to $0.9 \mathrm{nA}, 570 \mathrm{~ms}$ ) was shifted to $90 \mathrm{~ms}$ after the light flash to ensure action potential discharge during the visual-evoked inputs. 


\section{Group 3: single stimulus then light}

Single cortical stimulations were paired with light flashes at a delay of $250 \mathrm{~ms}$ ( 113 to 173 pulses, $0.2 \mathrm{~Hz}$ ). To aid occasional spike discharge in response to the stimulation, constant positive current was applied in two experiments. In contrast to all other groups, test stimuli before and after the plasticity protocol were applied at a fixed interval of 5-s independent of the neuron's membrane potential.

\section{Group 4: pre-post pairing then light}

Single cortical stimulations ( 60 to 108 pulses, $0.2 \mathrm{~Hz}$ ) were paired with a short intracellular current step (0.8 to $2 \mathrm{nA}, 5$ to $10 \mathrm{~ms})$ at a delay of 5 to $10 \mathrm{~ms}$, ideally inducing one action potential, and followed by a light flash at $250 \mathrm{~ms}$.

In three pre-post pairing experiments, BIC was ejected outside the SC $(n=1)$ or not ejected at all $(n=2)$. These experiments were excluded from group $4(n=10)$ and were used as a control for BIC-mediated visual responsiveness of subcortical visual pathways. In all other experiments, LFP recordings from the SC confirmed visual activation post BIC.

\section{HISTOLOGY}

In all experiments except for one rejected from group 4, tips of BIC ejection pipettes were verified to be within the SC using light microscopy of unstained sections or sections stained with cresyl violet $(0.1 \%)$. During recordings of at least $40 \mathrm{~min}$, neurons were passively filled with biocytin. Vibratome sections $(50 \mu \mathrm{m})$ containing biocytin-filled neurons were processed using standard histological procedures (Horikawa and Armstrong, 1988) and labeled cells were identified by fluorescent microscopy.

\section{DATA ANALYSIS}

Data was analyzed offline using MATLAB 7.1 with Signal Processing 6.4 and Statistics 5.1 Toolboxes. Axon binary files were imported into MATLAB using a function written by John Bender (http:// www.mathworks.de/matlabcentral/fileexchange/).

\section{Assessment of membrane potential fluctuations}

The membrane potential of all neurons at each time point was corrected offline by the estimated tip potential present at the time of recording. The distribution of the membrane potentials was assessed by all-amplitude histograms of intracellular recording over single trials of 5 to 10 -s duration. In order to visualize changes in membrane potential fluctuations over time, histograms of successive trials were concatenated and plotted as color-coded distributions over time, where each bin on the $x$-axis represents intervals, depending on trial length, of either 5 or $10 \mathrm{~s}$ and the $y$-axis displays the membrane potential values. In Figures 3-5, the time on the $x$-axis is approximate because of gaps in the recording. To quantify stability of membrane potential fluctuations across experiments, we evaluated the last minute of the baseline and the first minute of the second 5-min period after the end of the pairing protocol. This second time point was chosen because it was close to the time of maximal change in PSP slopes and the EEG slow-wave activity had completely recovered to baseline levels in all experiments. Recordings were rejected if there was progressive deterioration of the membrane potential fluctuations or the current-voltage relation between blocks of $\sim 30$ stimulus trials.

\section{Assessment of changes in synaptic transmission}

Independent of stimulation modus, only episodes with an initial membrane potential below a defined threshold close to the modal Down state membrane potential were accepted for analysis. The threshold was set at the membrane potential one third of the way between the 10th and 90th percentiles of the membrane potentials for each trial. Additionally, the membrane potential was required to have returned to below this threshold within $150 \mathrm{~ms}$ after stimulus to ensure that the evoked PSP was not contaminated by a spontaneous Up state. Membrane potential-dependent effects on included PSP amplitudes were small (Figure 1C, compare the lower two averages, all contributing single trials fell below the threshold). This was confirmed by initial results using a more stringent criterion (1/5 of the way between the 10th and 90th percentiles of the membrane potentials; data not shown) that remained qualitatively unchanged.

The strength of synaptic transmission was measured as the PSP slope. A linear fit to the PSP was calculated for a window of 1-ms length sliding over the whole ascending phase of the PSP and the maximal value was recorded. This way, the non-NMDAmediated component of the early depolarization caused by directly activated synapses was measured (Herrling, 1984, 1985; Jiang and North, 1991).

The time difference between stimulation and the center of the $1 \mathrm{~ms}$-long linear fit of the maximum slope was measured as the slope latency. If the distribution of slope latencies contained more than one peak clearly separated from others by troughs, individual peaks were used to define discernable PSP components separated by times of adjoining troughs (Figure 1D). Each PSP component was examined separately for the whole experiment. A potentially confounding factor was that later PSP components could be influenced by the depolarization caused by preceding PSP components. Linear regression analysis of the $5 \mathrm{~min}$ baseline period showed that there was no significant interaction in 13 out of 17 PSP component pairs (data not shown). In only one of the other four cases, both PSP components were significantly altered after the pairing protocol ( $p<0.05$; Wilcoxon rank sum test). Except for this case, PSP components were treated as independent measurements.

\section{Statistical evaluation}

To test for changes induced in individual experiments, PSP slope values measured during the first $10 \mathrm{~min}$ after the end of the plasticity protocol were compared to baseline values ( $5 \mathrm{~min}$ before plasticity protocol start) using a Wilcoxon rank sum test. For group analysis, means of PSP slopes recorded over 5 -min periods were normalized to baseline. Differences from baseline were tested for significant deviation from zero using a two-tailed student's $t$-test. To test for a timing dependent effect, PSP components were grouped in 3 ms-wide bins to include sufficient data points for statistical evaluation. With this or any smaller bin width, the relationship described by a polynomial fit (see below) was maintained in the binned means. For inter-group comparisons, a two-tailed student's $t$-test for comparison between two samples was used. The significance level was set to $p=0.05$. Unless noted, all data presented consists of mean \pm SEM. 


\section{Assessment of postsynaptic spike pattern during pairing protocol}

The percentage of trials with spikes was calculated from the ratio of trials including at least one spike during the 30 -ms time window following the cortico-striatal stimulus and the total number of trials of the pairing protocol. The percentage of trials with doublets was calculated accordingly from the ratio of trials including at least two spikes. The latency to spike was defined as the time difference between the time of the stimulus onset and the peak of the first spike.

The post-light spike rate was defined as the mean spike number within a 1-s window following the light flash, thus, capturing all spikes elicited during the relatively long visual-evoked Up states (Schulz et al., 2009).

\section{Regression models}

The data of potentially correlated parameters were fitted by a linear least-square fit to test for a significant interaction. To compensate for the spread of post-light spike rates over several orders of magnitude (from 0.1 to $>20 \mathrm{~Hz}$ ), these values were log-transformed before the linear regression analysis. To visualize the complex nature of spike-timing dependent plastic changes in PSP components, a 5th order polynomial was fitted to the data. This relationship could not be tested statistically because of the limited data set. The binned means at -3 and $3 \mathrm{~ms}$ were close to the maximum and the minimum of the polynomial fit at about -2.5 and $2.5 \mathrm{~ms}$, respectively, delimiting the linear portion of the polynomial fit. The values included in the binned means at $-3,0$ and $3 \mathrm{~ms}$ (i.e. -4.5 to $4.5 \mathrm{~ms}$ ) were tested for a significant linear correlation. Using narrower windows, e.g. -3 to $3 \mathrm{~ms}$, yielded $R^{2}$ values similar to those shown in Figure 6B, demonstrating that the result of the fit was not an accidental outcome of the chosen window width. In all other cases, no indications for a non-linear relationship between parameters were apparent from visual inspection of scatter plots.

\section{RESULTS}

All striatal neurons included in this study (stable for $>35 \mathrm{~min}$; $n=27$ ) were identified as SPNs by established electrophysiological criteria (Wilson and Kawaguchi, 1996; Mahon et al., 2000; Reynolds and Wickens, 2000), and verified in four experiments by morphology (Figure 1). Stimulation of the contralateral motor cortex elicited cortico-striatal PSPs, the amplitude of which was dependent

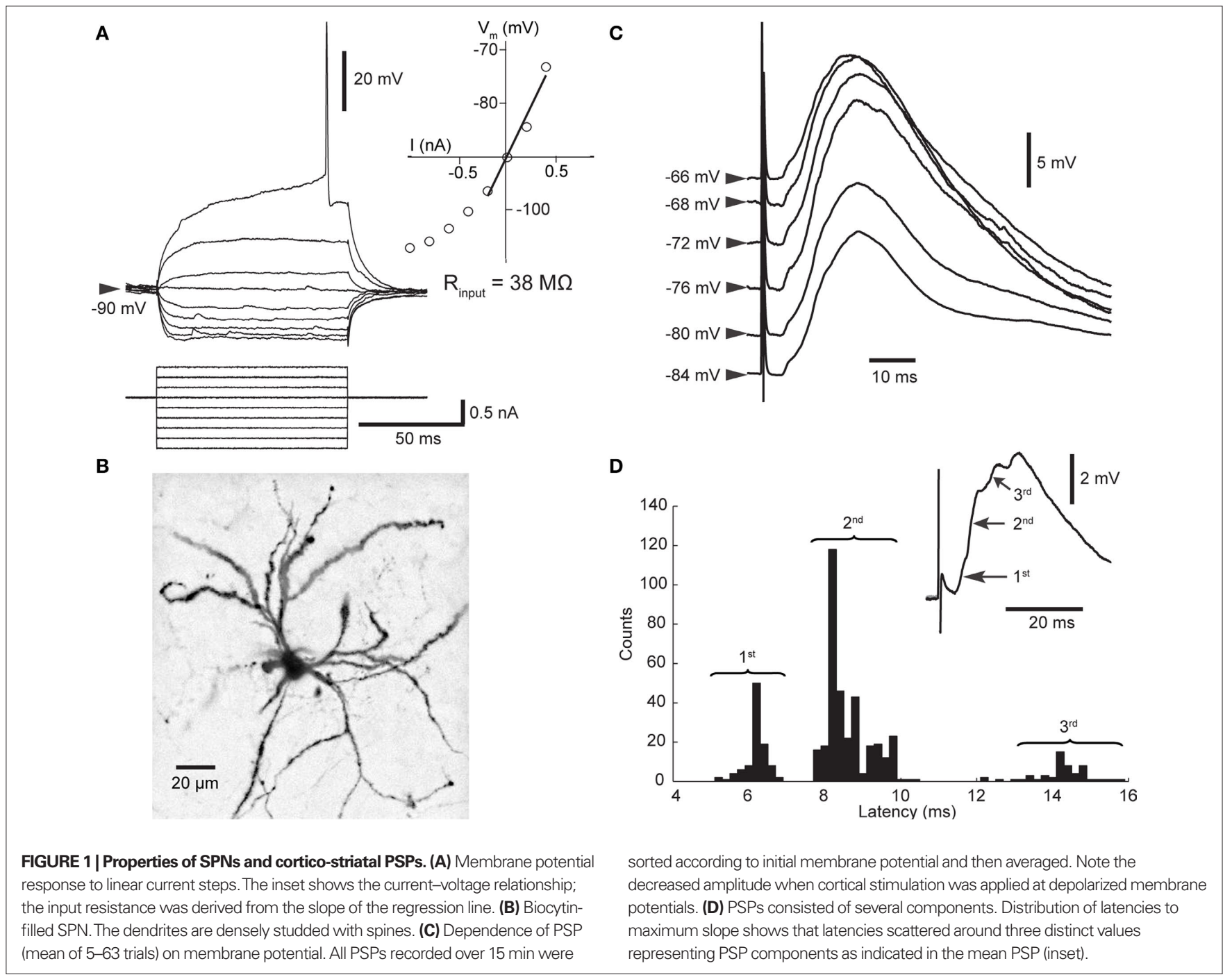


on the neuron's membrane potential (Figure 1C). Besides the voltage-dependent contribution of NMDA receptor-mediated currents (Ding et al., 2008; Pomata et al., 2008), voltage-dependent potassium conductances responsible for outward rectification likely contributed to this observation (Nisenbaum and Wilson, 1995). Note that the maximum potential of the evoked PSP changed little when evoked at depolarized membrane potentials (Figure 1C); therefore, changes in the amplitude of evoked PSPs do not necessarily represent synaptic plastic changes. Two strategies were used to minimize the influence of voltage sensitive conductance changes on the measurement of synaptic strength. Firstly, analysis of PSPs was restricted to those evoked during the SPN's more hyperpolarized Down state. Secondly, the maximum slope of the PSP rather than the amplitude was measured. Interestingly, the distribution of slope latencies exhibited two or three maxima in the majority of experiments (Figure 1D). Individual maxima represented different components of the PSP, probably mediated by distinct pathways. This observation was of particular interest in experiments investigating STDP.

\section{EFFECTS OF BIC-MEDIATED DISINHIBITION}

Disinhibition of subcortical visual pathways by local ejection of BIC into the SC caused a transient disruption of the Down to Up state membrane potential transitions typically observed in SPNs, as previously described (Schulz et al., 2009). Time-resolved membrane potential recordings, however, demonstrate that changes in PSP slopes we report here were not caused by shifts in the SPN's membrane potential activity (see Figures 3-5, panels labeled 'a'). As apparent in Table 1, all SPNs returned to Down state-Up state fluctuations comparable to baseline after the pairing protocol. Critically, the Down state membrane potential remained unchanged $(p>0.1$, paired $t$-test, $n=27$ neurons).

\section{EFFECTS OF VISUAL-EVOKED INPUTS IN COMBINATION WITH CORTICAL HFS}

During BIC-mediated disinhibition of subcortical visual pathways, cortical HFS was repetitively paired with a light flash to the contralateral eye. As the HFS was not sufficient to reliably induce postsynaptic spike discharge (Figure 2B), an intracellular current step was applied simultaneously to ensure spike discharge (Figure 2A). This protocol did not induce any changes in cortico-striatal synaptic strength during the first 10 -min post $(p>0.2$ for all five neurons; Wilcoxon rank sum test; Figure 3A). For the group, the normalized PSP slopes increased to $109.2 \pm 7.6 \%$ at 10 -min post, although this elevation was not significantly different from baseline $(p=0.29$; $t$-test, $n=5, t=1.21$ ).

A previous study induced strong and lasting potentiation in the cortico-striatal pathway when a suprathreshold current step was applied simultaneously to HFS of the substantia nigra pars compacta (Reynolds et al., 2001). To test the requirement of spike discharge during the phasic dopamine signal for synaptic potentiation, the same current step was applied delayed to the HFS, shortly after the light flash (Figure 2B). However, the presence of an increased post-light spike rate resulted in a significant depression during the first 10 min post in two SPNs $(p<0.05$; Wilcoxon rank sum test; Figure 3B), and a near-significant depression $(p=0.061)$ in a third. For the group, the normalized PSP slopes decreased to $82.7 \pm 2.4 \%$ at $5 \mathrm{~min}$ and $88.4 \pm 5.9 \%$ at $10 \mathrm{~min}$ post before returning to baseline values. In comparison with the previous HFS protocol, it was revealed that spike firing induced by a current step during the period of time at which the visual response was present significantly reduced PSP slopes at $5 \mathrm{~min}(p=0.014 ; t$-test, $n 1=5$, $n 2=4, t=3.25$; Figure 6A).

These results suggest that our HFS protocol was unable to induce significant potentiation of the cortico-striatal pathway even when consistently followed by visual-evoked subcortical inputs. This may have resulted from a restricted number of pairings (nominally 6) with the visual-evoked inputs. Thus, we next tested a protocol involving repetitive pairing of single cortical stimuli with light flashes.

\section{EFFECTS OF VISUAL-EVOKED INPUTS AND SINGLE CORTICAL STIMULI}

The effect of the repetitive pairing of single cortical stimulations $(\sim 120 ; 0.2 \mathrm{~Hz})$ followed $250 \mathrm{~ms}$ later by a light flash to the contralateral eye was tested in five SPNs. The number of spikes elicited during the protocol varied greatly between neurons, as evidenced from the percentage of trials with spikes (Table 2). The pairing protocol induced significant synaptic depression in three SPNs

Table 1 | Properties of SPNs before and after the plasticity protocols.

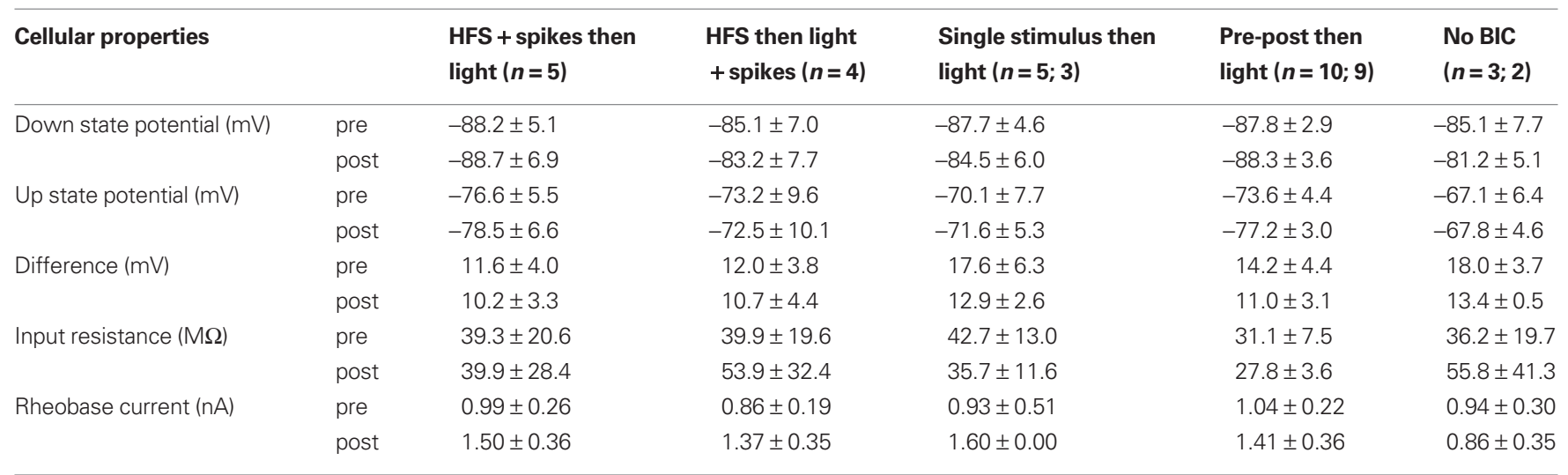

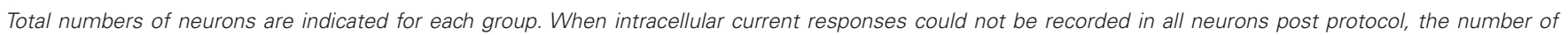
experiments included is represented by the second $n$ value. Data are mean \pm S.D. across neurons. 

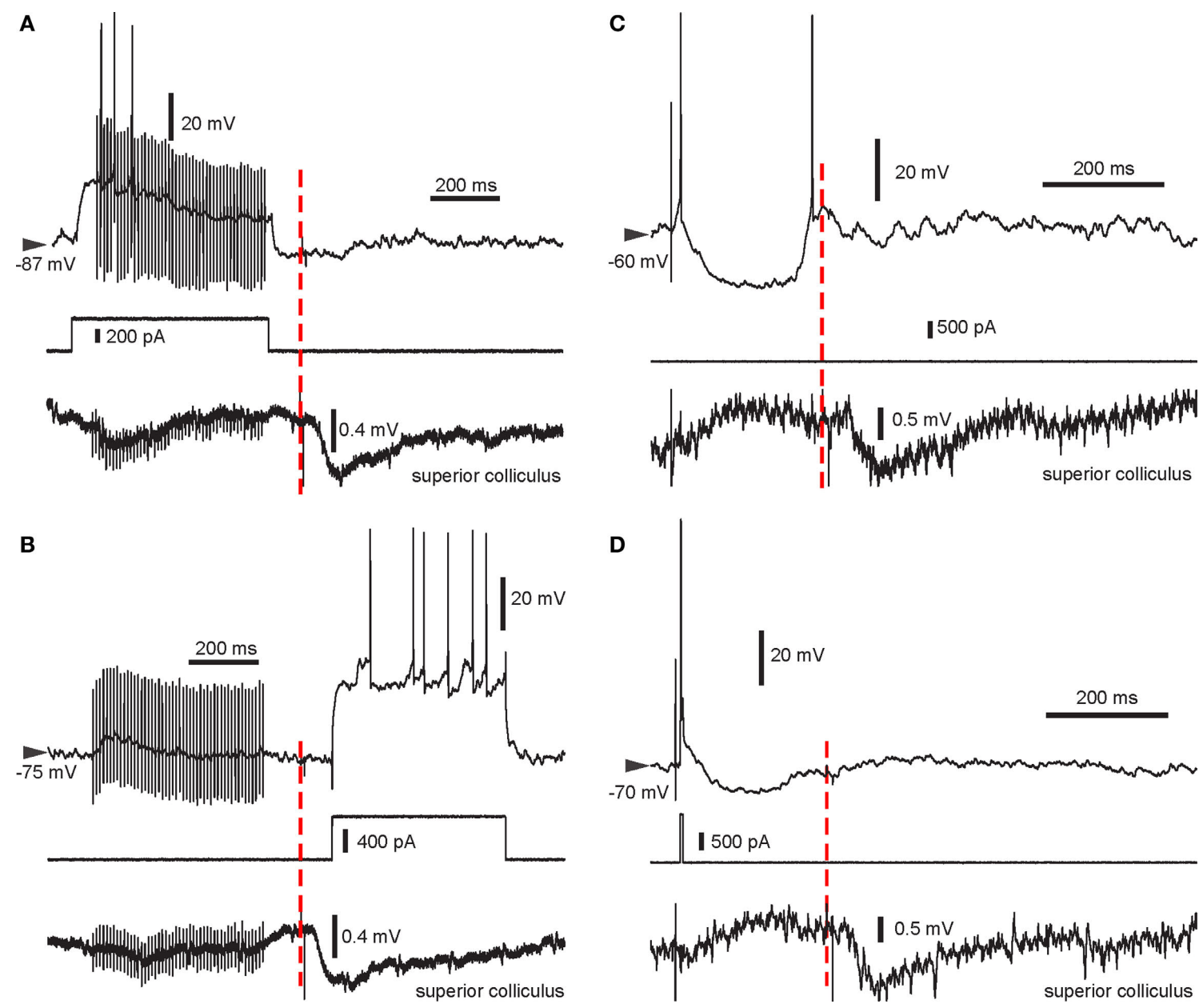

FIGURE 2 | Example traces from all four pairing protocols used. Intracellular recording (top trace), intracellular current injection (middle) and LFP recorded in the SC (bottom) are shown. The start of the light flash is indicated by the red dashed line. Note the negative deflection in the LFP recording indicating the VEP enabled by BIC. (A) HFS + spikes then light. (B) HFS then light + spikes.

( $p<0.05$; Wilcoxon rank sum test), even when the percentage of trials with spikes was increased by constant current injection. However, one SPN showed a tendency towards increased PSP slopes (08812n2). In this case, the pairing protocol involved a much higher percentage of trials with spikes than in all other experiments (61.9\%). To determine whether a high percentage of trials with spikes would favor the induction of potentiation, a further set of experiments was conducted with spikes consistently induced by a short current pulse.

\section{EFFECTS OF VISUAL-EVOKED INPUTS AND PRE-POST SPIKE PAIRING}

In 13 experiments, a short current pulse ( 5 to $10 \mathrm{~ms},+0.8$ to $+2 \mathrm{nA}$ ) was applied during the pairing protocol at a latency of 5-10 ms after the cortico-striatal stimulus. This regularly resulted in trials with spikes in $90.9 \pm 14.3 \%$ of pairing trials (mean \pm SD; range: 49.1-100\%). The mean latency across experiments to the evoked spike ranged from 8 to $15 \mathrm{~ms}$ after cortical stimulation. In three experiments without BIC ejection into the SC, no significant change of the PSP slope could be detected ( $p>0.1$; Wilcoxon rank sum test). In experiments involving BIC-mediated disinhibition of the SC $(n=10)$, significant changes in the PSP slope were induced in four experiments ( $p<0.05$; Wilcoxon rank sum test), with two showing significant increases and two showing a decrease despite the similarity of the applied pairing protocol. In three of these cases (shown in Figures 4 and 5), the change in PSP slope appeared relatively stable and persisted for $>20 \mathrm{~min}$.

\section{FACTORS CONTRIBUTING TO VARIABLE PLASTICITY OUTCOME}

A number of factors were evaluated to determine if they contributed to the variability in plasticity results between SPNs. In regression analyses, the normalized PSP slope at 10-20 min post was not correlated to stimulus strength, initial PSP slope at baseline, VEP amplitude recorded from the SC, or number of pairings during the plasticity-inducing protocol (all: $p>0.1, n=10$ ). This suggested that variation of experimental parameters was an unlikely explanation. 


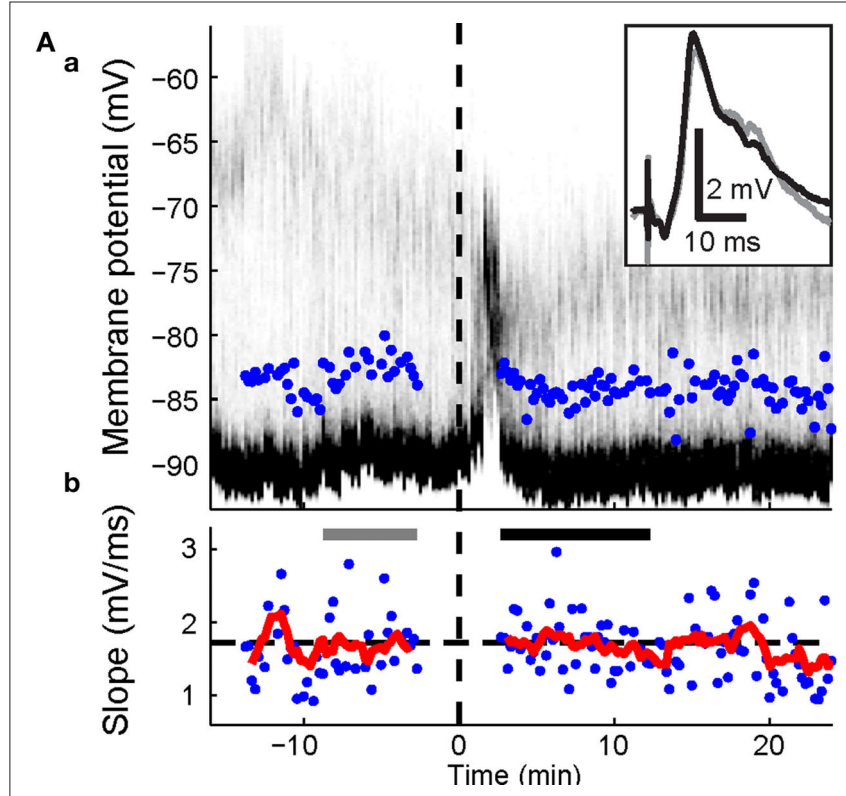

FIGURE 3 | Effects of visual-evoked inputs in combination with corticostriatal HFS. Panels a, time-resolved membrane potential distribution. Gray-scale indicates the probability for the neuron to be at respective membrane potential ( $y$-axis); black depicts a high probability, white a low probability. Time on the $x$-axis is given in relation to BIC ejection (dotted line at 0). Maximum amplitude of PSPS are indicated. Panels b, maximal slopes of PSP. Dashed line indicates mean PSP

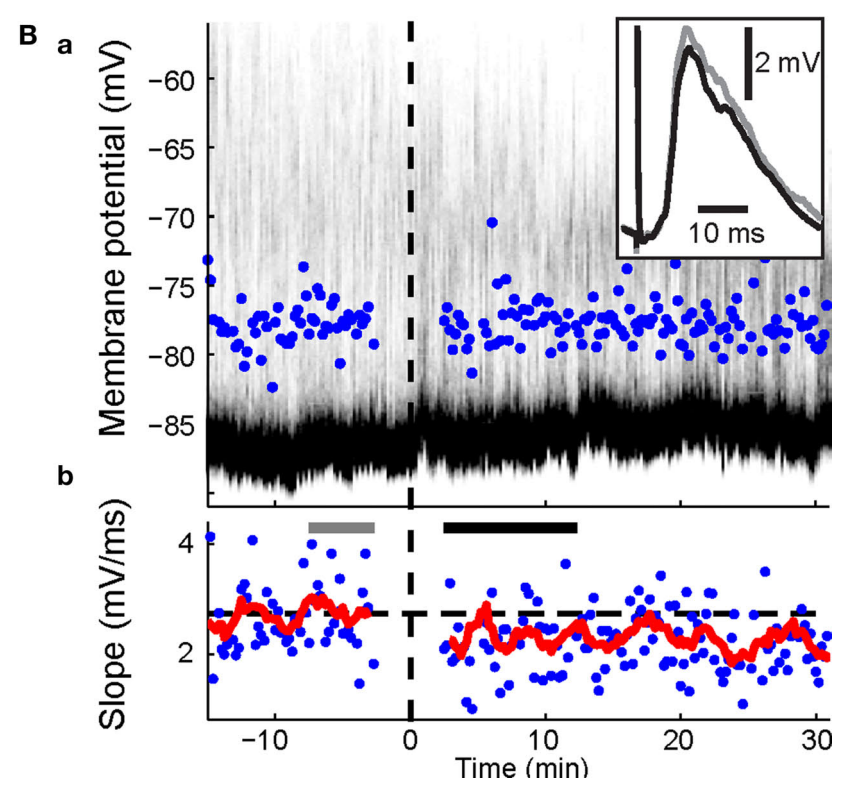

slopes at baseline. Running average of 9 consecutive values is indicated (red trace). PSP traces (top right inset in a) are mean PSPs recorded in the time indicated by the gray and black bars, respectively. (A) Representative example of cortico-striatal HFS with current-evoked spike discharge followed by light. No significant changes were induced. (B) HFS followed by light with current-evoked spike discharge induced depression ( $p=0.002$; Wilcoxon rank sum test).

Table 2 | Summary of results from pairing single cortical stimuli followed by light.

\begin{tabular}{|c|c|c|c|c|c|c|}
\hline & & \multicolumn{5}{|c|}{ Individual experiments } \\
\hline & & $08701 n 1$ & $09811 n 1$ & $08604 n 2$ & $09721 n 1$ & $08812 n 2$ \\
\hline \multirow[t]{2}{*}{ PSP slope (mV/ms) } & pre & $2.20 \pm 0.10$ & $2.43 \pm 0.06$ & $2.27 \pm 0.11$ & $2.39 \pm 0.08$ & $2.48 \pm 0.10$ \\
\hline & post & $1.76 \pm 0.05$ & $1.97 \pm 0.05$ & $1.96 \pm 0.05$ & $2.26 \pm 0.06$ & $2.68 \pm 0.10$ \\
\hline Percentage of baseline & & $(80.0 \%)$ & $(81.1 \%)$ & (86.3\%) & $(94.6 \%)$ & $(108 \%)$ \\
\hline$p$-value & & $<0.001$ & $<0.001$ & 0.010 & 0.231 & 0.097 \\
\hline Current (nA) & & - & - & +0.1 & +0.4 & - \\
\hline No pairings & & 120 & 121 & 116 & 173 & 113 \\
\hline Trials with spikes (\%) & & 0 & 25.6 & 31.9 & 13.9 & 61.9 \\
\hline Trials with doublets (\%) & & 0 & 0 & 0 & 0 & 7.1 \\
\hline Post-light spike rate $(\mathrm{Hz})$ & & 0 & 0.74 & 3.49 & 0.37 & 1.02 \\
\hline
\end{tabular}

p-values are from Wilcoxon rank sum tests of single trial data with approximately 30 trials per period.

\section{Postsynaptic spiking}

Number and timing of postsynaptic spikes were examined for effects on the plasticity outcome. There was no significant correlation between normalized PSP slope at 10 to 20 -min post and the percentage of trials with spikes or the mean spike latency $(p>0.1$, $n=10)$. The short current pulse (5-10 ms) occasionally induced multiple spikes (3.0-63.3\% of trials; $n=5$ neurons) and postsynaptic high-frequency spike bursts may have been more efficient for inducing potentiation than single spikes (Pike et al., 1999; Kampa et al., 2006). However, no significant correlation between plasticity outcome and percentage of trials with doublets could be found $(p>0.1, n=10)$. Therefore, there appeared to be no statistically significant influence on the PSP slope at 10-20 min, from the number of postsynaptic spikes following the cortical stimulation.

Results from the HFS experiments suggested that an elevated spike rate during the visual-evoked inputs could potentially bias the plasticity outcome towards depression. Three SPNs in the prepost pairing group fired spikes during the second following light in at least some trials. One SPN exhibited a particularly high postlight spike rate of $4.55 \mathrm{~Hz}$ (Figure 4A). Ten to twenty minutes of post pairing protocol, this neuron exhibited a significant decrease of the PSP slope from $1.40 \pm 0.09$ to $1.05 \pm 0.05 \mathrm{mV} / \mathrm{ms}(75 \%$ of baseline; $p<0.001$; Wilcoxon rank sum test, $n 1=40, n 2=55$, 

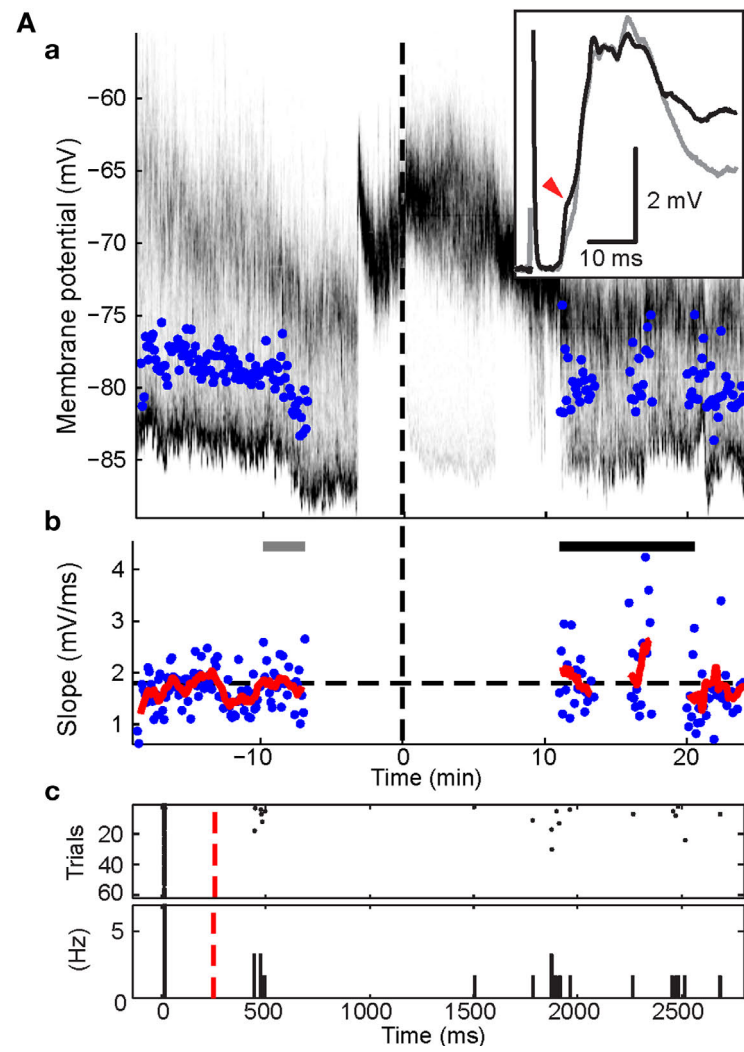

FIGURE 4 | Effects of post-light spike rate on cortico-striatal plasticity in experiments involving pre-post pairing. Format for panels $a$ and $b$ is the same as in Figure 3. Panels c, raster plot and peri-stimulus time histogram of spikes during the pairing protocol. Cortical stimulus was applied at 0 , the intracellular current pulse at approximately $10 \mathrm{~ms}$, and light at $250 \mathrm{~ms}$

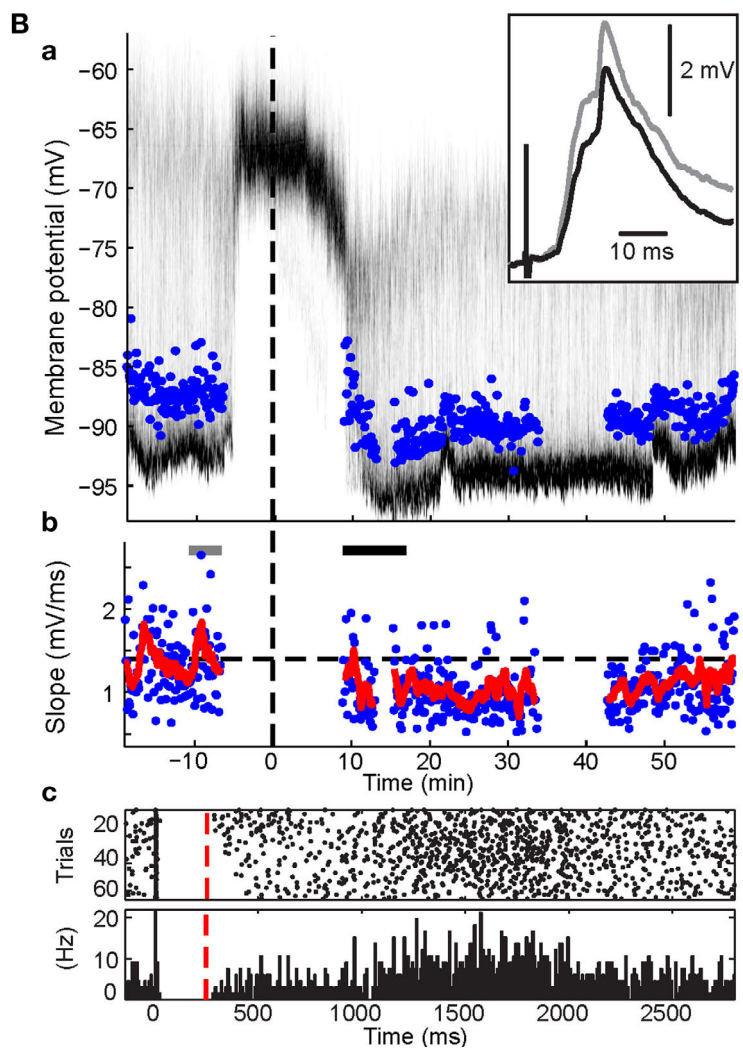

(red dashed line). (A) Example recording showing depression $(p<0.001$; Wilcoxon rank sum test). Note the high spike rate post light. (B) Example recording showing selective increases in a PSP component (red arrow head; $p<0.05$; Wilcoxon rank sum test) without a significant change of the overall PSP slope. rank sum $=2407.5)$. In contrast, synaptic potentiation of either a single PSP component (Figure 4B) or the whole PSP (Figure 5A) was found in the other two cases after the pairing protocol when the post-light spike rate was 0.1 and $0.13 \mathrm{~Hz}$, respectively. Across experimental groups, increasing log-transformed post-light spike rates were highly correlated with decreased normalized PSP slopes following the pairing protocol $\left(R^{2}=0.55, p=0.009, n=11\right.$ active neurons, $F=11.04$; Figure 6D). These observations supported the hypothesis that post-light spike rates modulated the plasticity outcome and provided an explanation for the depression in one pre-post pairing experiment.

\section{Presynaptic factors}

The observation that PSPs regularly consisted of up to three distinct components suggested that evoked presynaptic spikes reached the postsynaptic striatal SPN via multiple axon projection trajectories, potentially involving several mono- and di-synaptic pathways (Wilson, 1986, 1987; Reiner et al., 2003; Lei et al., 2004). If pathways were affected differently by the plasticity protocol, then the relative contribution of individual pathways to the evoked PSP would have determined the plasticity outcome. Therefore, all identified PSP components were analyzed individually. There were no indications for a relationship between latencies and normalized PSP slope at
10-20 min (Figure 6Bb) and 20-30 min post (data not shown). Thus, a pathway-specific effect was an unlikely explanation of the observed variability in plasticity outcomes.

\section{Interaction between pre- and postsynaptic activation}

An alternative explanation was that the precise timing between pre- and postsynaptic spikes influenced the plasticity outcomes (Figure 5). To test this hypothesis, normalized slopes of PSP components were plotted against the time difference between PSP component latency and the first postsynaptic spike (Figure 6Bc). Latencies of individual PSP components were expected to follow closely the timing of the presynaptic spike at the synapses that caused the inputs. Therefore, PSP component latencies represented a more meaningful reference time than the time of the electrical stimulus to the contralateral cortex. This analysis revealed that PSP components with a latency equal to or up to $4.5 \mathrm{~ms}$ slower than the mean latency to spike (i.e. negative time-to-spike points in Figure $6 \mathrm{Bc}$, plotted as $3 \mathrm{~ms}$ bins at $-3 \mathrm{~ms}$ and $0 \mathrm{~ms}$ in Figure 6Bd) always showed a tendency to increase after the pairing protocol. At 10 to $20 \mathrm{~min}$ after the protocol, PSP components within $1.5 \mathrm{~ms}$ either side of spike latency (plotted at $0 \mathrm{~ms}$ in Figure 6Bd) were significantly potentiated $(115.2 \pm 3.9 \% ; p=0.011 ; t$-test, $n=6, t=3.93)$. In contrast, PSP components preceding the spike (i.e. positive time-to-spike 


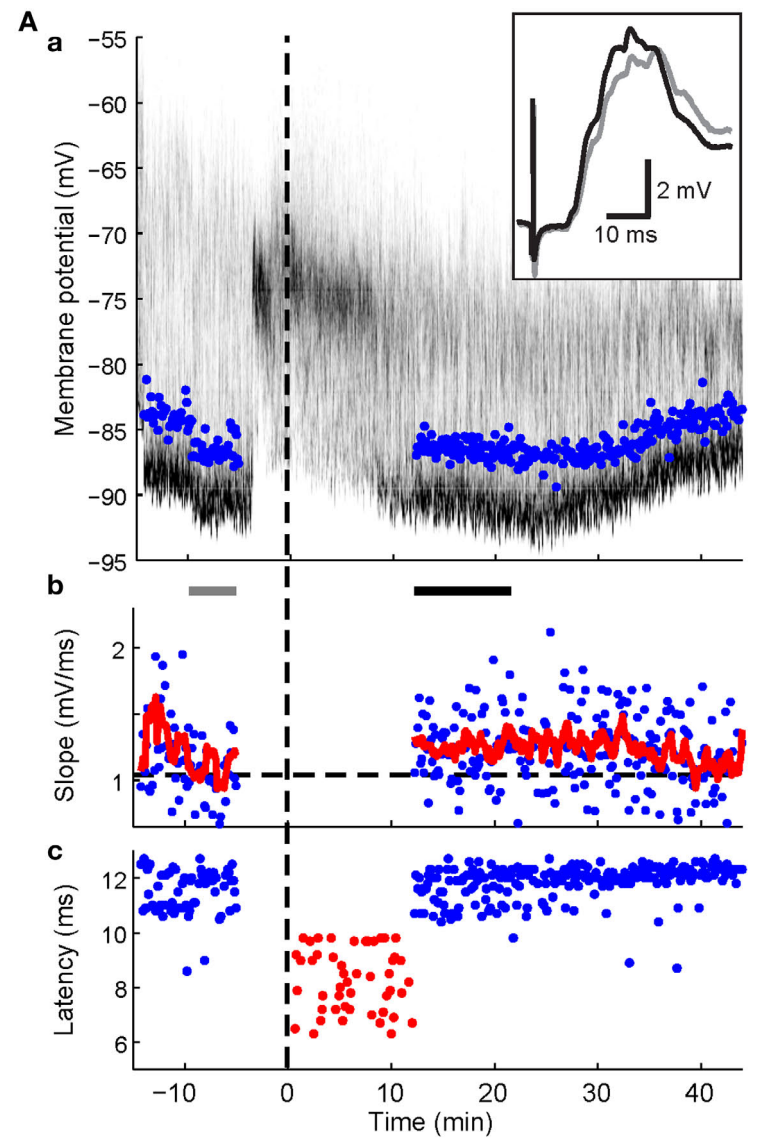

FIGURE 5 | Spike-timing-dependent increases and decreases in corticostriatal synaptic efficacy. Format for panels $a$ and $b$ is the same as in Figure 3 Panels c, latencies of maximal slope measurements (blue dots) and spike times during the plasticity protocol (red dots). (A) Example recording showing potentiation ( $p<0.001$; Wilcoxon rank sum test) after pairing PSPs with a

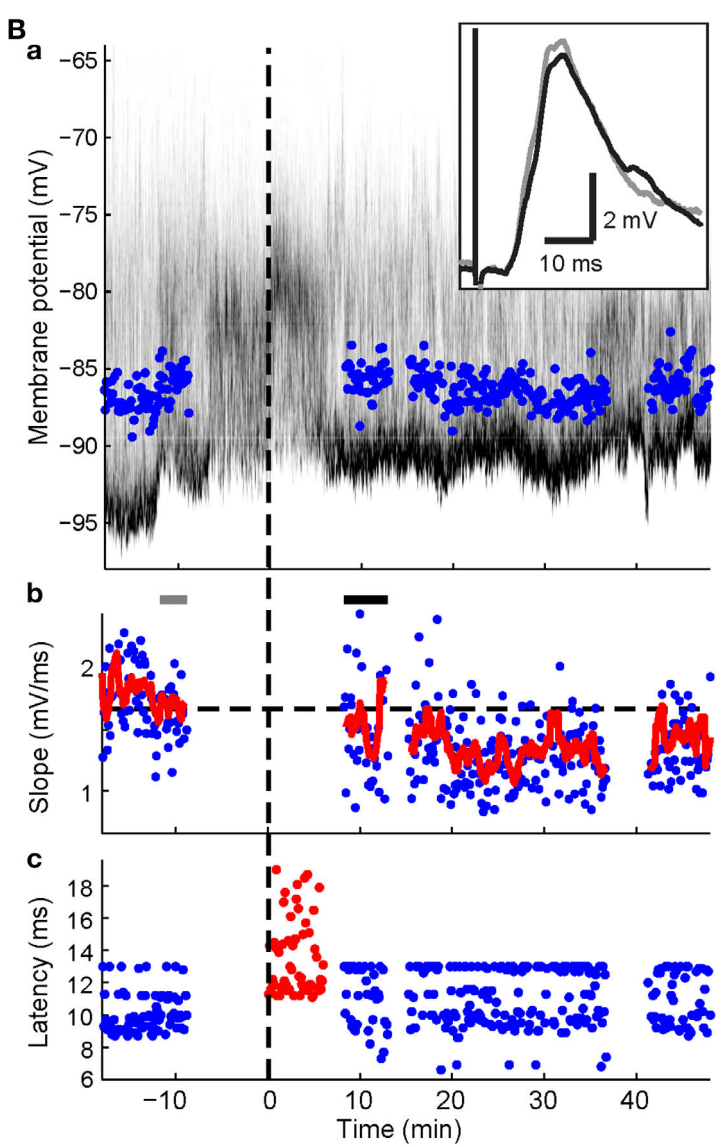

postsynaptic spike elicited by somatic current injection. Note spike latencies were relatively early compared to maximal slope latencies. (B) Example recording showing depression ( $p=0.038$; Wilcoxon rank sum test) after pairing with current-evoked spikes following the maximal PSP slope. Note the relative higher frequency of later latency components after the protocol. points in Figure 6Bc) showed a higher variability but a tendency towards depression. At 20-30 min post, PSP components between 1.5 and $4.5 \mathrm{~ms}$ before the spike (plotted at $+3 \mathrm{~ms}$ in Figure 6Bd) were significantly decreased $(86.5 \pm 4.5 \% ; p=0.024$; $t$-test, $n=7$, $t=-3.0)$. Thus, at 10-20 min after the protocol, synaptic potentiation appeared to be favored for most PSP components, however, over the ensuing 10-min period, a more balanced picture of potentiation of components following the spike but depression of components prior to the spike emerged (Figure 6Bc). Spike-timing and induced synaptic changes of PSP components between -4.5 and $4.5 \mathrm{~ms}$ to spike latency were significantly negatively correlated for both time points following the protocol ( $p<0.05, n=16$ PSP components; Figure 6Bd). However, in the absence of BIC-mediated disinhibition of the SC, PSP components remained largely unchanged and were not subject to STDP (Figure 6C).

In summary, the precise temporal relationship between individual PSP components and the evoked spike appeared to be the determining factor for the direction of the induced plasticity and thus accounted for a large proportion of the variability between experiments involving BIC-mediated disinhibition of subcortical visual pathways.

\section{DISCUSSION}

The aim of the present investigation was to test the effect of a more physiological procedure for evoking dopamine release into the striatum (Dommett et al., 2005) on the outcome of established synaptic plasticity protocols at the cortico-striatal synapse. The results showed that HFS protocols did not induce significant potentiation under these conditions. Consistent pre-post pairing induced significant changes in at least one PSP component in every experiment that involved disinhibition of the SC. However, the direction of change exhibited a high variability. This was attributed to the relative timing between pre- and postsynaptic spikes on a scale of a few milliseconds. The discussion will focus on this unexpected outcome and its implications for synaptic plasticity in vivo.

\section{CORTICO-STRIATAL STDP}

The direction of change induced by pre-post pairing depended on the relative timing of the PSP component relative to the spike. The phenomenon of STDP at the cortico-striatal synapse has been described in previous in vitro studies (Fino et al., 2005; Pawlak and Kerr, 2008). However, the characteristics of the underlying temporal rule remains controversial. Thus, Fino et al. (2005) found that repeti- 

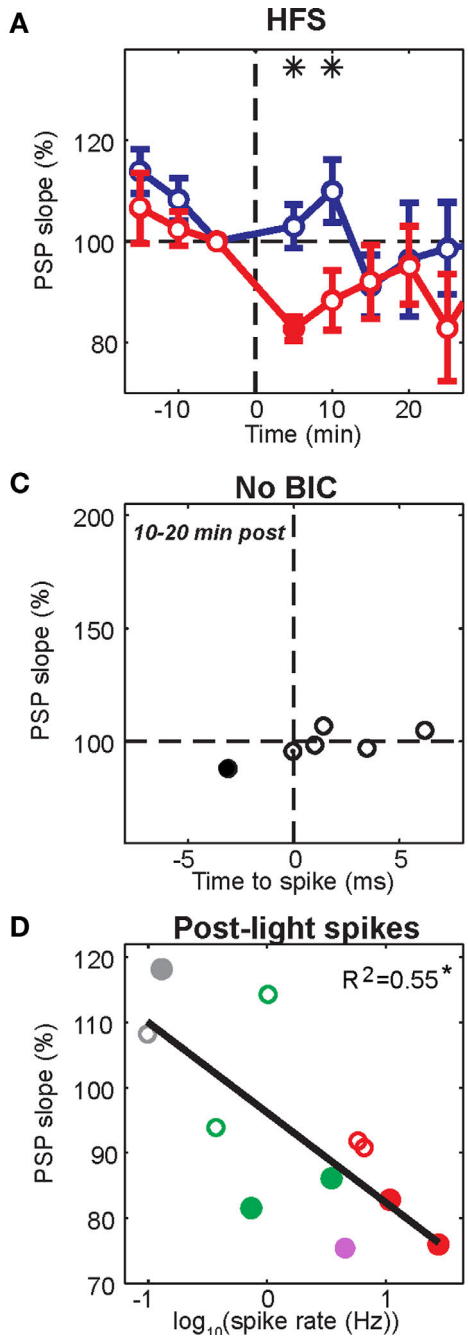

B

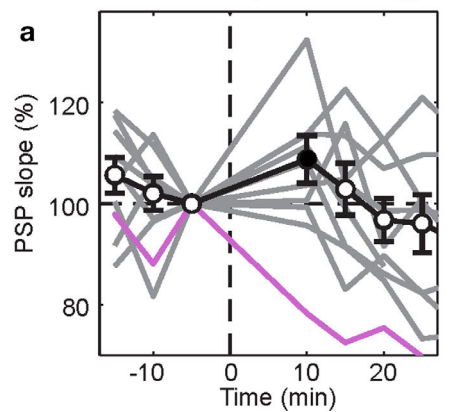

C

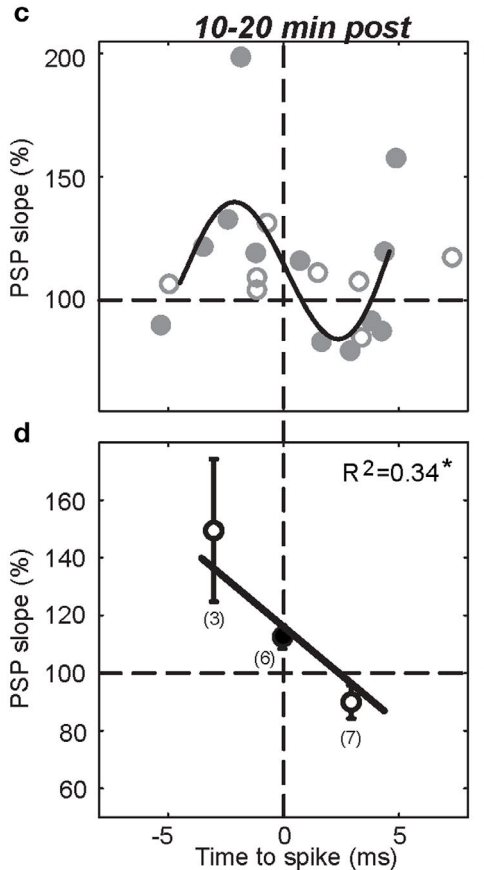

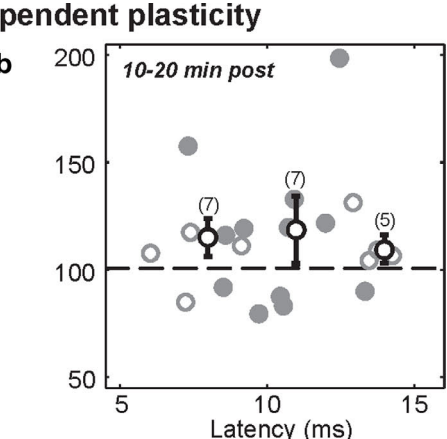

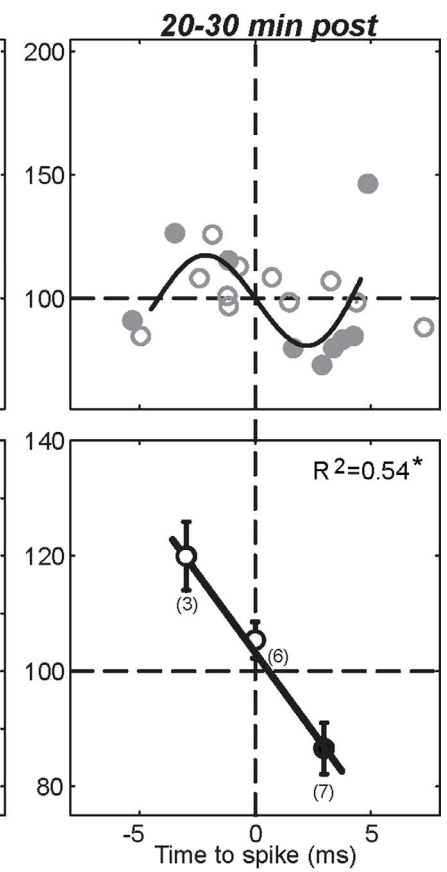

FIGURE 6 | Summary of cortico-striatal plasticity after visual activation of subcortical pathways. In all panels, significant changes from baseline are indicated by filled circles. (A) Effects of HFS on normalized PSP slope. Current-evoked spike discharge during visual inputs (red, $n=4$ ) resulted in depression (asterisks; $p<0.05, t$-test) when compared to current-evoked spike discharge during HFS (blue, $n=5$ ). (B) Panel a, pre-post pairing resulted in variable outcomes across experiments (gray traces). One SPN (purple; same as Figure 4A) was excluded because of a high post-light spike rate. Panels b-d, analysis of individual PSP components. Means (black) of binned data (3-ms bins; number of included points in brackets) indicate that changes did not depend on latency (B, panel b) but on relative timing to evoked spike (B, panel d). Although the true relationship between spike-timing and synaptic change was complex (approximated by a 5 th order polynomial fit in $\mathbf{B}$, panel $c$ ), there was a significant linear correlation for spike-timing values between -4.5 and $4.5 \mathrm{~ms}(p<0.05$, $n=16$; $\mathbf{B}$, panel d). (C) PSP components remained largely unchanged in experiments without BIC-mediated disinhihibition of the SC $(n=3)$. (D) Significant effect of post-light spike rate on normalized PSP slope 10-20 min post across experimental groups $(p=0.009)$. Color-code is the same as in $(\mathbf{A}-\mathbf{C})$; green represents pairing of visual and single cortical stimuli without a postsynaptic current step. tive pre-post pairing induced depression, while post-pre pairing induced potentiation. This is the reverse of results by Pawlak and Kerr (2008) and the established rule for most intracortical excitatory synapses (Markram et al., 1997; Sjostrom et al., 2001; Froemke and Dan, 2002). Protocols used by the two groups exhibit two potentially important differences: Fino et al. (2005) stimulated the cortex at $1 \mathrm{~Hz}$ during the pairing protocol; in contrast, Pawlak and Kerr (2008) maintained the stimulation frequency at $0.1 \mathrm{~Hz}$ throughout the experiment; however, their experiments were performed in the presence of the $\mathrm{GABA}_{\mathrm{A}}$ receptor antagonist picrotoxin. In the present study, we used a pairing protocol ( 60 pairings at $0.2 \mathrm{~Hz}$ ) very similar to the one used by Pawlak and Kerr (2008). On the other hand, the
SPNs were recorded in vivo where all GABAergic inhibition was completely intact. Using this approach, we found synaptic changes of similar magnitude $( \pm 20 \%)$ to those observed by Pawlak and Kerr (2008) during the first $20 \mathrm{~min}$ after the end of the pairing protocol. However, in most experiments synaptic modifications followed a much more stringent timing rule in vivo and affected only single components rather than the whole PSP. Note that the experiments were designed to induce potentiation by near coincident pre-and postsynaptic activation. Yet, synaptic inputs preceding the spike by about $3 \mathrm{~ms}$ were depressed. In contrast, PSP components exactly coinciding with or closely following the postsynaptic spike by about $3 \mathrm{~ms}$ were potentiated (an impressive example is shown in Figure 
5A). Components of the PSP outside of this narrow time window remained on average unaffected by the protocol. Thus, STDP in vivo appears to require a relative temporal order that is closer to the rules proposed by Fino et al. (2005), albeit on a contracted time scale. We will next consider how in vivo background activity and synaptic inhibition may have contributed to these observations.

\section{STDP IN VIVO}

To our knowledge, this is the first study using electrical stimulus-evoked PSPs for an STDP protocol in a vertebrate in vivo. Previous in vivo studies on STDP used sensory evoked PSPs with much more complex pre- and postsynaptic activation patterns and were performed in young or developing neural systems (Meliza and Dan, 2006; Mu and Poo, 2006; Jacob et al., 2007). Most relevant to the current study, Jacob et al. (2007) restricted whisker-evoked PSPs to subthreshold amplitudes and paired them with 1-2 currentevoked spikes. Under these conditions, pre-post pairing induced a significant increase in amplitude but not in slope in most neurons (Jacob et al., 2007), indicating that non-synaptic mechanisms like dendritic plasticity may have contributed (Campanac and Debanne, 2008). However, when significant increases or decreases in slopes were induced by pre-post and post-pre pairing, respectively, the effects were of a magnitude $( \pm 20 \%)$ similar to the present study (Jacob et al., 2007). Further support for a reduced efficacy of STDP in vivo comes from in vitro studies employing the dynamic clamp technique (Delgado and Desai, 2008, 2009) in order to simulate the high-conductance state of neurons in vivo (Destexhe et al., 2003). Under these conditions, windows for efficient modulation of synaptic transmission strength were greatly reduced to less than $10 \mathrm{~ms}$ and the magnitude of induced changes was also reduced to levels comparable with the present study. Interestingly, $\beta$-adrenergic receptor activation could increase the induced potentiation (Delgado and Desai, 2009). These results show striking parallels to the present study: STDP does not appear to be a very effective mechanism under more physiological conditions, unless an additional neuromodulatory signal boosts its effect.

Mechanistically, less effective STDP under in vivo conditions could be explained by attenuated back-propagation of spikes. However, calcium transients evoked by spike invasion of dendrites appear to be a pre-requisite for STDP (Kampa et al., 2006). Potassium conductances in particular have been shown to significantly attenuate back-propagation of spikes (Hoffman et al., 1997). The prominent potassium conductances during the Up state in SPNs (Nisenbaum and Wilson, 1995) likely impede action potential back-propagation (Day et al., 2008). Accordingly, spontaneous action potentials in SPNs in organotypic cultures elicit the largest calcium transient in dendrites when they are evoked early in the Up state (Kerr and Plenz, 2004).

GABA inputs can also impair spike back-propagation (Tsubokawa and Ross, 1996; Xiong and Chen, 2002). In the present study, spike back-propagation was most likely subject to inhibitory modulation. The striatum is a structure that consists almost exclusively of inhibitory GABAergic neurons, most prominently of SPNs that have extensive local axon arborizations on neighboring SPNs (Wilson and Groves, 1980). In the same animal, cortical stimulation at parameters that elicited large-amplitude PSPs in one SPN often failed to induce a sizeable PSP in another SPN only a few hundred microns away (data not shown). This suggests that some striatal neurons close to the neuron recorded from were likely to be more strongly activated, including occasional spike discharge. These coincident inhibitory inputs would have hampered effective spike back-propagation (Tsubokawa and Ross, 1996; Xiong and Chen, 2002) and therefore the induction of potentiation. This could potentially explain the curious observation that PSP components preceding a spike by more than 2 ms were regularly depressed: the same PSP component could already have caused a spike in neighboring cells and the evoked inhibitory inputs onto the recorded neuron prevented an effective invasion of the dendrites by the spike. Two distinct signaling mechanisms underlying potentiation and depression after an STDP protocol have recently been described for the cortico-striatal synapse (Venance et al., 2009). Using the close timing of pre- and postsynaptic activation in the present study, we likely activated both mechanisms simultaneously. Then, simultaneous inhibitory inputs may have selectively hampered the NMDA-dependent mechanism for the induction of potentiation by locally clamping the membrane potential below the depolarization required for the removal of the magnesium block. It is intriguing that the study by Fino et al. (2005) that originally described depression induced by pre-post pairing was performed in the absence of GABA antagonists. However, a reversed STDP window has also been described for Purkinje-like GABAergic neurons (Bell et al., 1997). Similarly, cortical and other GABAergic interneurons exhibit at least partially reversed STDP windows (Tzounopoulos et al., 2004; Lu et al., 2007). Further work is therefore needed to clarify the specific contributions of cell-intrinsic versus competitive inhibitory mechanisms in regulating the STDP window at the cortico-striatal synapse.

\section{EFFECTS OF CORTICO-STRIATAL HFS}

High-frequency stimulation protocols have been widely applied in striatal preparations and regularly result in synaptic depression rather than potentiation (Calabresi et al., 2007; Surmeier et al., 2007). However, simultaneously increased levels of dopamine prevent depression and promote potentiation in vitro and in vivo (Wickens et al., 1996; Reynolds and Wickens, 2000). Accordingly, the results from group 1 (HFS + spikes then light) are very similar to results from a group of SPNs in a previous in vivo study that received contralateral HFS plus intracellular current and simultaneous low-frequency stimulation of the substantia nigra pars compacta (Reynolds and Wickens, 2000). This would indicate that dopamine is able to block depression even if it is released shortly after the HFS. It is important to note, however, that the HFS protocol was apparently not sufficient to induce stronger potentiation than the STDP protocol. It is possible that the number of pairings of cortico-striatal inputs with the visual stimulus was insufficient. Visual-evoked dopamine release is expected to be much smaller than if the substantia nigra was stimulated directly at high frequency. In previous in vivo studies using the same design of six 500-ms stimulation trains, robust potentiation was only induced if stimulation to the substantia nigra pars compacta was applied at high frequency, but not if it was applied at low frequency (Reynolds and Wickens, 2000; Reynolds et al., 2001). This suggests that a particularly large dopamine transient during each pairing is necessary to induce potentiation if the pairing number is low. 


\section{IMPACT OF POSTSYNAPTIC SPIKING AFTER THE LIGHT FLASH}

Interestingly, SPNs that were driven to a high spike rate after the light flash showed a strong tendency towards depression of the preceding cortico-striatal inputs across experimental groups. Assuming this reflects a functionally meaningful mechanism, synaptic plasticity rules in the striatum would appear to prevent potentiation of synaptic weights at SPNs if they are also activated at the time of a different set of inputs. This mechanism could promote segregation of movements, cues and outcome representations by SPNs during learning (Barnes et al., 2005).

In conclusion, the present study has demonstrated that synaptic plasticity can, in principle, be induced using visual-evoked subcortical inputs, very likely including dopamine. However, potentiation could only be induced in a subset of single-spike pairing experiments involving many repetitions. In contrast to common expectations, post-pre pairing on a millisecond-timescale promoted potentiation, whereas pre-post pairing promoted depression. Additional in vivo studies are required to test the effectiveness of STDP and its regulation by neuromodulators in other brain areas in order to validate our present models of brain computations.

\section{ACKNOWLEDGMENTS}

This work was supported by the Marsden Fund of the Royal Society of New Zealand (John N. J. Reynolds), and Wellcome Trust grant WT080943 (Peter Redgrave). Jan M. Schulz received a University of Otago Postgraduate Scholarship.

\section{REFERENCES}

Barnes, T. D., Kubota, Y., Hu, D., Jin, D. Z. Z., and Graybiel, A. M. (2005). Activity of striatal neurons reflects dynamic encoding and recoding of procedural memories. Nature 437, 1158-1161.

Bell, C. C., Han, V. Z., Sugawara, Y., and Grant, K. (1997). Synaptic plasticity in a cerebellum-like structure depends on temporal order. Nature 387, 278-281.

Calabresi, P., Picconi, B., Tozzi, A., and Di Filippo, M. (2007). Dopaminemediated regulation of corticostriatal synaptic plasticity. Trends Neurosci. 30, 211-219.

Campanac, E., and Debanne, D. (2008). Spike timing-dependent plasticity: a learning rule for dendritic integration in rat CA1 pyramidal neurons. J. Physiol. (Lond.) 586, 779-793.

Coizet, V., Comoli, E., Westby, G. W. M., and Redgrave, P. (2003). Phasic activation of substantia nigra and the ventral tegmental area by chemical stimulation of the superior colliculus: an electrophysiological investigation in the rat. Eur. J. Neurosci. 17, 28-40.

Comoli, E., Coizet, V., Boyes, J., Bolam, J. P., Canteras, N. S., Quirk, R. H., Overton, P.G., and Redgrave, P. (2003). A direct projection from superior colliculus to substantia nigra for detecting salient visual events. Nat. Neurosci. 6, 974-980.

Day, M., Wokosin, D., Plotkin, J. L., Tian, X. Y., and Surmeier, D. J. (2008). Differential excitability and modulation of striatal medium spiny neuron dendrites. J. Neurosci. 28, 11603-11614.

Delgado, J. Y., and Desai, N. S. (2008). In vivo-like conductances limit spiketiming dependent plasticity. Abstr. Soc. Neurosci. 39, 40.43

Delgado, J. Y., and Desai, N. S. (2009). In vivo-like conductance levels control the induction of spike-timing dependent plasticity. Abstr. - Soc. Neurosci $40,41.48$.
Destexhe, A., Rudolph, M., and Pare, D. (2003). The high-conductance state of neocortical neurons in vivo. Nat. Rev. Neurosci. 4, 739-751.

Ding, J., Peterson, J. D., and Surmeier, D. J. (2008). Corticostriatal and thalamostriatal synapses have distinctive properties. J. Neurosci. 28, 6483-6492.

Dommett, E., Coizet, V., Blaha, C. D., Martindale, J., Lefebvre, V., Walton, N., Mayhew, J. E., Overton, P. G., and Redgrave, P. (2005). How visual stimuli activate dopaminergic neurons at short latency. Science 307, 1476-1479.

Erro, M. E., Lanciego, J. L., and GimenezAmaya, J. M. (2002). Re-examination of the thalamostriatal projections in the rat with retrograde tracers. Neurosci. Res. 42, 45-55.

Fino, E., Glowinski, J., and Venance, L. (2005).Bidirectional activity-dependent plasticity at corticostriatal synapses. $J$. Neurosci. 25, 11279-11287.

Froemke, R. C., and Dan, Y. (2002). Spiketiming-dependent synaptic modification induced by natural spike trains. Nature 416, 433-438.

Herrling, P. L. (1984). Evidence that the cortically evoked EPSP in cat caudate neurons is mediated by non-NMDA excitatory amino-acid receptors. $J$. Physiol. (Lond.) 353, P98-P98.

Herrling, P. L. (1985). Pharmacology of the corticocaudate excitatory postsynaptic potential in the cat: evidence for its mediation by quisqualate- or kainate-receptors. Neuroscience 14, 417-426.

Hoffman, D. A., Magee, J. C., Colbert, C. M., and Johnston, D. (1997). $\mathrm{K}^{+}$channel regulation of signal propagation in dendrites of hippocampal pyramidal neurons. Nature 387, 869-875.

Horikawa, K., and Armstrong, W. E. (1988). A versatile means of intracellular labeling: Injection of biocytin and its detection with avidin conjugates. $J$. Neurosci. Methods 25, 1-11.

Izhikevich, E. M. (2007). Solving the distal reward problem through linkage of
STDP and dopamine signaling. Cereb. Cortex 17, 2443-2452.

Jacob, V., Brasier, D. J., Erchova, I. Feldman, D., and Shulz, D. E. (2007) Spike timing-dependent synaptic depression in the in vivo barrel cortex of the rat. J. Neurosci. 27, 1271-1284.

Jiang, Z. G., and North, R. A. (1991) Membrane properties and synaptic responses of rat striatal neurones in vitro. J. Physiol. 443, 533-553.

Kampa, B. M., Letzkus, J. J., and Stuart, G. J. (2006). Requirement of dendritic calcium spikes for induction of spiketiming-dependent synaptic plasticity. J. Physiol. (Lond.) 574, 283-290.

Kerr, J. N., and Plenz, D. (2004). Action potential timing determines dendritic calcium during striatal up-states. $J$. Neurosci. 24, 877-885.

Kerr, J. N., and Wickens, J. R. (2001) Dopamine D1/D5 receptor activation is required for long-term potentiation in the rat neostriatum in vitro. J. Neurophysiol. 85, 117-124.

Lei, W. L., Jiao, Y., Del Mar, N., and Reiner, A. (2004). Evidence for differential cortical input to direct pathway versus indirect pathway striatal projection neurons in rats. J. Neurosci. 24 8289-8299.

Lu, J. T., Li, C. Y., Zhao, J. P., Poo, M. M. and Zhang,X.H. (2007). Spike-timingdependent plasticity of neocortical excitatory synapses on inhibitory interneurons depends on target cell type. J. Neurosci. 27, 9711-9720.

Mahon, S., Delord, B., Deniau, J. M., and Charpier, S. (2000). Intrinsic properties of rat striatal output neurones and timedependent facilitation of cortical inputs in vivo. J. Physiol. 527(Pt 2), 345-354.

Markram, H., Lubke, J., Frotscher, M., and Sakmann, B. (1997). Regulation of synaptic efficacy by coincidence of postsynaptic APs and EPSPs. Science 275, 213-215.

McGeorge, A. J., and Faull, R. L. (1989) The organization of the projection from the cerebral cortex to the striatum in the rat. Neuroscience 29,503-537.
Meliza, C. D., and Dan, Y. (2006). Receptive-field modification in rat visual cortex induced by paired visual stimulation and single-cell spiking. Neuron 49, 183-189.

Minamimoto, T., Hori, Y., and Kimura, M. (2005). Complementary process to response bias in the centromedian nucleus of the thalamus. Science 308 1798-1801.

Mu, Y., and Poo, M. M. (2006). Spike timing-dependent LTP/LTD mediates visual experience-dependent plasticity in a developing retinotectal system. Neuron 50, 115-125.

Nisenbaum, E. S., and Wilson, C. J. (1995). Potassium currents responsible for inward and outward rectification in rat neostriatal spiny projection neurons. J. Neurosci. 15, 4449-4463.

Pawlak, V., and Kerr, J. N. D. (2008) Dopamine receptor activation is required for corticostriatal spiketiming-dependent plasticity. $J$ Neurosci. 28, 2435-2446.

Pike, F. G., Meredith, R. M., Olding, A. W. A., and Paulsen, O. (1999). Postsynaptic bursting is essential for 'Hebbian' induction of associative long-term potentiation at excitatory synapses in rat hippocampus. $J$. Physiol. (Lond.) 518, 571-576.

Pomata, P. E., Belluscio, M. A., Riquelme, L.A., and Murer, M. G. (2008). NMDA receptor gating of information flow through the striatum in vivo. J. Neurosci. 28, 13384-13389.

Redgrave, P., Gurney, K., and Reynolds, J. (2008). What is reinforced by phasic dopamine signals? Brain Res. Rev. 58, 322-339.

Reiner,A., Jiao, Y., Del Mar, N., Laverghetta, A.V., and Lei, W. L. (2003). Differential morphology of pyramidal tract-type and intratelencephalically projectingtype corticostriatal neurons and their intrastriatal terminals in rats. J. Comp. Neurol. 457, 420-440.

Reynolds, J. N. J., Hyland, B. I., and Wickens, J. R. (2001). A cellular 
mechanism of reward-related learning. Nature 413, 67-70.

Reynolds, J. N. J., and Wickens, J. R. (2000). Substantia nigra dopamine regulates synaptic plasticity and membrane potential fluctuations in the rat neostriatum, in vivo. Neuroscience 99 , 199-203.

Reynolds, J. N. J., and Wickens, J. R. (2003). A state-dependent trigger for electrophysiological recording at predetermined membrane potentials. J. Neurosci. Methods 131, 111-119.

Schulz, J. M., Redgrave, P., Mehring, C., Aertsen, A., Clements, K. M., Wickens, J. R., and Reynolds, J. N. J. (2009). Short-latency activation of striatal spiny neurons via subcortical visual pathways. J. Neurosci. 29, 6336-6347.

Shen, W. X., Flajolet, M., Greengard, P., and Surmeier, D. J. (2008). Dichotomous dopaminergic control of striatal synaptic plasticity. Science 321, 848-851.

Sjostrom,P.J.,Turrigiano, G.G., and Nelson, S. B. (2001). Rate, timing, and cooperativity jointly determine cortical synaptic plasticity. Neuron 32, 1149-1164.

Surmeier, D. J., Ding, J., Day, M., Wang, Z. F., and Shen, W. X. (2007). D1 and
D2 dopamine-receptor modulation of striatal glutamatergic signaling in striatal medium spiny neurons. Trends Neurosci. 30, 228-235.

Tsubokawa, H., and Ross, W. N. (1996). IPSPs modulate spike backpropagation and associated $\left[\mathrm{Ca}^{2+}\right] \mathrm{i}$ changes in the dendrites of hippocampal CA1 pyramidal neurons. J. Neurophysiol. 76, 2896-2906.

Tzounopoulos, T., Kim, Y., Oertel, D., and Trussell, L. O. (2004). Cell-specific, spike timing-dependent plasticities in the dorsal cochlear nucleus. Nat. Neurosci. 7, 719-725.

Venance, L., Paille, V., Cui, Y., MoreraHerreras, T., Deniau, J.-M., and Fino, E. (2009). Two coincidence detectors are required for the corticostriatal spike timing-dependent plasticity. Abstr. - Soc. Neurosci. 40, 41.42.

Wickens, J. R., Begg, A. J., and Arbuthnott, G. W. (1996). Dopamine reverses the depression of rat corticostriatal synapses which normally follows highfrequency stimulation of cortex in vitro. Neuroscience 70, 1-5.

Wickens, J. R., Budd, C. S., Hyland, B. I., and Arbuthnott, G. W. (2007a). Striatal contributions to reward and decision making - Making sense of regional variations in a reiterated processing matrix. Ann. N. Y. Acad. Sci.1104, 192-212.

Wickens, J. R., Horvitz, J. C., Costa, R. M., and Killcross, S. (2007b). Dopaminergic mechanisms in actions and habits. J. Neurosci. 27, 8181-8183.

Wilson, C. J. (1986). Postsynaptic potentials evoked in spiny neostriatal projection neurons by stimulation of ipsilateral and contralateral neocortex. Brain Res. 367, 201-213.

Wilson, C. J. (1987). Morphology and synaptic connections of crossed corticostriatal neurons in the rat. J. Comp. Neurol. 263, 567-580.

Wilson, C. J., and Groves, P. M. (1980). Fine structure and synaptic connections of the common spiny neuron of the rat neostriatum: a study employing intracellular inject of horseradish peroxidase. J. Comp. Neurol. 194, 599-615.

Wilson, C. J., and Kawaguchi, Y. (1996). The origins of two-state spontaneous membrane potential fluctuations of neostriatal spiny neurons. J. Neurosci. 16, 2397-2410.
Xiong, W. H., and Chen, W. R. (2002). Dynamic gating of spike propagation in the mitral cell lateral dendrites. Neuron 34, 115-126.

Conflict of Interest Statement: The authors declare that research was conducted in the absence of any commercial or financial relationships that could be construed as a potential conflict of interest.

Received: 14 February 2010; paper pending published: 23 April 2010; accepted: 31 May 2010; published online: 02 July 2010.

Citation: Schulz JM, Redgrave $P$ and Reynolds JNJ (2010) Cortico-striatal spike-timing dependent plasticity after activation of subcortical pathways. Front. Syn. Neurosci. 2:23. doi: 10.3389/ fnsyn.2010.00023

Copyright (c) 2010 Schulz, Redgrave and Reynolds. This is an open-access article subject to an exclusive license agreement between the authors and the Frontiers Research Foundation, which permits unrestricted use, distribution, and reproduction in any medium, provided the original authors and source are credited. 